\title{
Ideal Fermion Delocalization in Higgsless Models
}

\author{
R. Sekhar Chivukula and Elizabeth H. Simmons \\ Department of Physics and Astronomy, Michigan State University \\ East Lansing, MI 48824, USA \\ E-mail: sekhar@msu.edu, esimmons@msu.edu
}

\author{
Hong-Jian He \\ Department of Physics, University of Texas \\ Austin, TX 78712, USA \\ E-mail: hjhe@physics.utexas.edu
}

\author{
Masafumi Kurachi and Masaharu Tanabashi \\ Department of Physics, Tohoku University \\ Sendai 980-8578, Japan \\ E-mail: kurachi@tuhep.phys.tohoku.ac.jp, tanabash@tuhep.phys.tohoku.ac.jp
}

\begin{abstract}
In this note we examine the properties of deconstructed Higgsless models for the case of a fermion whose $S U(2)$ properties arise from delocalization over many sites of the deconstructed lattice. We derive expressions for the correlation functions and use these to establish a generalized consistency relation among correlation functions. We discuss the form of the $W$ boson wavefunction and show that if the probability distribution of the delocalized fermions is appropriately related to the $W$ wavefunction, then deviations in precision electroweak parameters are minimized. In particular, we show that this "ideal fermion delocalization" results in the vanishing of three of the four leading zero-momentum electroweak parameters defined by Barbieri, et. al. We then discuss ideal fermion delocalization in the context of two continuum Higgsless models, one in Anti-deSitter space and one in flat space. Our results may be applied to any Higgsless linear moose model with multiple $S U(2)$ groups, including those with only a few extra vector bosons.
\end{abstract}

KEYwords: Dimensional Deconstruction, Electroweak Symmetry Breaking, Higgsless Theories, Delocalization. 


\section{Introduction}

Higgsless models [1] incorporate a mechanism for electroweak symmetry breaking without a conventional scalar Higgs particle. The most popular models [2, 3] are based on a fivedimensional $S U(2) \times S U(2) \times U(1)$ gauge theory in a slice of Anti-deSitter space, and electroweak symmetry breaking is encoded in the boundary conditions of the gauge fields on this space. The resulting spectrum includes the massless photon, the $W$ and $Z$ bosons (which are the first Kaluza-Klein excitations of the five-dimensional gauge fields), and an infinite tower of additional massive vector bosons (the remaining "KK" excitations). The unitarity of longitudinal $W$ and $Z$ boson scattering is ensured by the exchange of these other heavy vector bosons [4, 5, 6, 7], rather than through the exchange of a scalar Higgs boson [8]. There have been numerous studies of electroweak properties and collider phenolmenology [9, 10, 11, 12, 13, 14, 15, 16] in the context of these five-dimensional models.

An alternative approach to analyzing the properties of Higgsless models [17, 18, 19, 20, 21, 22, 23, 24] is to use deconstruction [25, 26] and to compute the electroweak parameters $\alpha S$ and $\alpha T$ [27, 28, 29] in a related linear moose model [30]. We have recently shown [24] how to compute all four of the leading zero-momentum electroweak parameters defined by Barbieri et. al. 15 in a very general class of linear moose models. Using deconstruction, we are able to relate the size of these electroweak corrections directly to the spectrum of the KK modes, which is constrained by unitarity. Taking the continuum limit, our results apply directly to models with arbitrary background 5-D geometry, spatially dependent gauge-couplings, and brane kinetic energy terms.

Using the deconstruction approach, we first studied Higgsless models with localized fermions, i.e. fermions which derive their $S U(2)$ and $U(1)$ properties from a single site on the deconstructed lattice. We found [24] that any model of this kind which does not have extra light vector bosons (with masses of order the $W$ and $Z$ ) cannot simultaneously satisfy unitarity bounds and the constraints of precision electroweak data. Our analyses also apply directly to a large class of models of extended electroweak symmetry which have only a few non-standard vector bosons [31, 32, 33]; these models are motivated in part by models of hidden local symmetry 34, 35, 36, 37, 38].

It has recently been proposed [39, 40] that the size of corrections to electroweak processes may be reduced by including delocalized fermions. In deconstruction, a delocalized fermion is realized as a fermion whose $S U(2)$ properties arise from several sites on the deconstructed lattice [41, 42]. We have previously considered in detail the case of a fermions whose $S U(2)$ properties arise from two adjacent sites [41], and have confirmed that (even in that simple case) it is possible to minimize the electroweak parameter $\alpha S$ by choosing a suitable amount of fermion delocalization.

In this work we examine in detail the properties of deconstructed Higgsless models for the case of a fermion whose $S U(2)$ properties arise from delocalization over many sites of the deconstructed lattice. We derive explicit expressions for the correlation functions and

use these to establish a generalized consistency relation among them. We discuss the form 


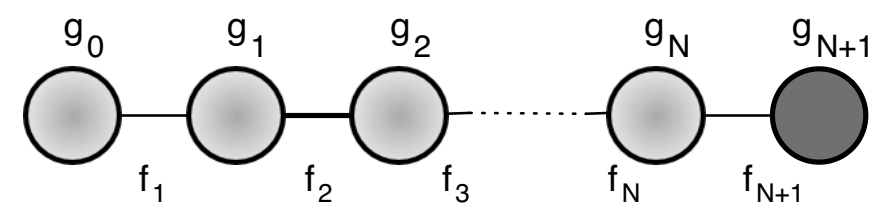

Figure 1: Moose diagram of the model analyzed in this note. Sites 0 to $N$ are $S U(2)$ gauge groups, site $N+1$ is a $U(1)$ gauge group. The fermions are delocalized in the sense that the $S U(2)$ couplings of the fermions arise (potentially) from the gauge groups at all sites from 0 to $N$. The $U(1)$ coupling comes from the gauge group at site $N+1$.

of the $W$ boson wavefunction and show that if the probability distribution of the delocalized fermions is appropriately related to the $W$ wavefunction, deviations in precision electroweak parameters are minimized. In particular, we show that this "ideal fermion delocalization" results in the vanishing of three of the four leading zero-momentum electroweak parameters defined by Barbieri, et. al. [15]. We then briefly discuss* ideal fermion delocalization in the context of two continuum Higgsless models, one in Anti-deSitter space and one in flat space. Our results may be applied to any Higgsless linear moose model with multiple $S U(2)$ groups, including those with only a few extra vector bosons.

\section{Review of the Model and Notation}

We study a deconstructed Higgsless model, as shown diagrammatically (using "moose notation" [30]) in fig. 1. The model incorporates an $S U(2)^{N+1} \times U(1)$ gauge group, and $N+1$ nonlinear $(S U(2) \times S U(2)) / S U(2)$ sigma models in which the global symmetry groups in adjacent sigma models are identified with the corresponding factors of the gauge group. The Lagrangian for this model at leading order is given by

$$
\mathcal{L}_{2}=\frac{1}{4} \sum_{j=1}^{N+1} f_{j}^{2} \operatorname{tr}\left(\left(D_{\mu} U_{j}\right)^{\dagger}\left(D^{\mu} U_{j}\right)\right)-\sum_{j=0}^{N+1} \frac{1}{2 g_{j}^{2}} \operatorname{tr}\left(F_{\mu \nu}^{j} F^{j \mu \nu}\right),
$$

with

$$
D_{\mu} U_{j}=\partial_{\mu} U_{j}-i A_{\mu}^{j-1} U_{j}+i U_{j} A_{\mu}^{j},
$$

where all gauge fields $A_{\mu}^{j}(j=0,1,2, \cdots, N+1)$ are dynamical. The first $N+1$ gauge fields $(j=0,1, \ldots, N)$ correspond to $S U(2)$ gauge groups; the last gauge field $(j=N+1)$ corresponds to the $U(1)$ gauge group. The symmetry breaking between the $A_{\mu}^{N}$ and $A_{\mu}^{N+1}$ follows an $S U(2)_{L} \times S U(2)_{R} / S U(2)_{V}$ symmetry breaking pattern with the $U(1)$ embedded as the $T_{3}$-generator of $S U(2)_{R}$. Our analysis proceeds for arbitrary values of the gauge couplings

${ }^{*}$ A detailed description of the deconstructed models corresponding in the continuum limit to ideally delocalized fermions in flat and warped space will be deferred to a subsequent work 43 . 
and $f$-constants. In the continuum limit, therefore, this allows for arbitrary background 5D geometry, spatially dependent gauge-couplings, and brane kinetic energy terms for the gauge-bosons.

All four-fermion processes, including those relevant for the electroweak phenomenology of our model, depend, respectively, on the neutral and charged gauge field propagator matrices

$$
D^{Z}\left(Q^{2}\right) \equiv\left[Q^{2} \mathcal{I}+M_{Z}^{2}\right]^{-1}, \quad D^{W}\left(Q^{2}\right) \equiv\left[Q^{2} \mathcal{I}+M_{W}^{2}\right]^{-1}
$$

Here, $M_{Z}^{2}$ and $M_{W}^{2}$ are, respectively, the mass-squared matrices for the neutral and charged gauge bosons and $\mathcal{I}$ is the identity matrix. Consistent with [20], $Q^{2} \equiv-q^{2}$ refers to the Euclidean momentum.

The neutral vector meson mass-squared matrix is of dimension $(N+2) \times(N+2)$

$$
M_{Z}^{2}=\frac{1}{4}\left(\begin{array}{c|c|c|c|c}
g_{0}^{2} f_{1}^{2} & -g_{0} g_{1} f_{1}^{2} & & & \\
\hline-g_{0} g_{1} f_{1}^{2} & g_{1}^{2}\left(f_{1}^{2}+f_{2}^{2}\right) & -g_{1} g_{2} f_{2}^{2} & & \\
& \ddots & \ddots & \ddots & \\
\hline & & -g_{N-1} g_{N} f_{N}^{2} & g_{N}^{2}\left(f_{N}^{2}+f_{N+1}^{2}\right) & -g_{N} g_{N+1}^{2} f_{N+1}^{2}
\end{array}\right) .
$$

and the charged current vector bosons' mass-squared matrix is the upper-left $(N+1) \times(N+1)$ dimensional block of the $M_{Z}^{2}$ matrix. The neutral mass matrix (2.4) is of a familiar form that has a vanishing determinant, due to a zero eigenvalue. Physically, this corresponds to a massless neutral gauge field - the photon. The non-zero eigenvalues of $M_{Z}^{2}$ are labeled by $\mathrm{m}_{Z z}^{2}(z=0,1,2, \cdots, N)$, while those of $M_{W}^{2}$ are labeled by $\mathrm{m}_{W w}^{2}(w=0,1,2, \cdots, N)$.

The lowest massive eigenstates corresponding to eigenvalues $\mathrm{m}_{Z 0}^{2}$ and $\mathrm{m}_{W 0}^{2}$ are, respectively, identified as the usual $Z$ and $W$ bosons. We will refer to these last eigenvalues by their conventional symbols $M_{Z}^{2}, M_{W}^{2}$; the distinction between these and the corresponding mass matrices should be clear from context. We will denote the eigenvectors corresponding to the photon, $Z$, and $W$ by $v_{i}^{\gamma}, v_{i}^{Z}$, and $v_{j}^{W}$. These eigenvectors are normalized as

$$
\sum_{i=0}^{N+1}\left(v_{i}^{\gamma}\right)^{2}=\sum_{i=0}^{N+1}\left(v_{i}^{Z}\right)^{2}=\sum_{j=0}^{N}\left(v_{j}^{W}\right)^{2}=1 .
$$

Inspection of the matrix $M_{Z}^{2}$ reveals that each component of the photon eigenvector is inversely related to the gauge coupling at the corresponding site

$$
v_{i}^{\gamma}=\frac{e}{g_{i}}, \quad \text { where } \quad \frac{1}{e^{2}}=\sum_{i=0}^{N+1} \frac{1}{g_{i}^{2}} .
$$

In the continuum limit, the eigenstates with masses $\mathrm{m}_{W w}^{2}$ and $\mathrm{m}_{Z z}^{2}$ correspond to the higher Kaluza-Klein ("KK") excitations of the five-dimensional $W$ and $Z$ gauge fields.

Generalizing the usual mathematical notation for "open" and "closed" intervals, we may denote [24] the neutral-boson mass matrix $M_{Z}^{2}$ as $M_{[0, N+1]}^{2}-i . e$. it is the mass matrix for the entire moose running from site 0 to site $N+1$ including the gauge couplings of both endpoint 
groups. Analogously, the charged-boson mass matrix $M_{W}^{2}$ is $M_{[0, N+1)}^{2}$ - it is the mass matrix for the moose running from site 0 to link $N+1$, but not including the gauge couping at site $N+1$. This notation will be useful in thinking about the properties of sub-matrices $M_{[0, i)}^{2}$ of the full gauge-boson mass matrices that arise in our discussion of fermion delocalization, and also the corresponding eigenvalues $\mathrm{m}_{i \hat{i}}^{2}(\hat{i}=1,2, \ldots, i)$. We will denote the lightest such eigenvalue $\mathrm{m}_{i 1}^{2}$ by the symbol $M_{i}^{2}$.

We will find it useful to define the following sums over heavy eigenvalues for phenomenological discussions:

$$
\Sigma_{Z} \equiv \sum_{z=1}^{N} \frac{1}{\mathrm{~m}_{Z z}^{2}}, \quad \Sigma_{W} \equiv \sum_{w=1}^{N} \frac{1}{\mathrm{~m}_{W w}^{2}}, \quad \Sigma_{[0, i)} \equiv \operatorname{Tr} M_{[0, i)}^{-2} .
$$

That is, $\Sigma_{Z}$ and $\Sigma_{W}$ are the sums over inverse-square masses of the higher neutral- and charged-current KK modes of the full model.

\section{Deconstructed Delocalized Fermions}

The authors of [39, 40] consider the possibility that the standard model fermions have wavefunctions with finite extent in the fifth dimension. In practice, this means that the observed fermions are the lightest eigenstates of five-dimensional fermions, just as the $W$ and $Z$ gaugebosons are the lightest in a tower of "KK" excitations. These authors show that by adjusting the five-dimensional wavefunction of the light fermions, one can modify (and potentially eliminate) the dangerously large corrections to precision electroweak measurements. In this section, we establish what we mean by fermion delocalization in a deconstructed model; we explore the consequences in subsequent sections.

\subsection{Deconstructing fermion delocalization}

The deconstructed version of fermion delocalization proceeds very similarly to the continuum version. Since a five-dimensional spinor is equivalent to a four-dimensional Dirac fermion, one introduces a separate Dirac fermion at each site (i.e. one left-handed and one right-handed Weyl spinor per site, $\psi_{L}^{i}$ and $\psi_{R}^{i}$ ) on the interior of the moose diagram of fig. 1. The chirality of the standard model fermions is introduced by adjusting the boundary conditions for the fermion fields at the ends of the moose. A convenient choice 44] (consistent with the weak interactions) that we will adopt corresponds to

$$
\psi_{L}^{N+1}=0, \quad \psi_{R}^{0}=0
$$

Discretizing the Dirac action for a five-dimensional fermion in an arbitrary background metric then corresponds to introducing site-dependent masses $\left(m_{j}\right)$ for the Dirac fermions at each interior site and postition-dependent Yukawa interactions $\left(y_{j}\right)$ which couple the left-handed modes at site $j$ to the right-handed modes at site $j+1$

$$
\mathcal{L}_{5 f}=-\sum_{j=1}^{N-1} m_{j} \bar{\psi}_{L}^{j} \psi_{R}^{j}-\sum_{j=0}^{N-1} f_{j+1} y_{j+1}\left(\overline{\psi_{L}^{j}} U_{j+1} \psi_{R}^{j+1}\right)+\text { h.c. },
$$


where gauge-invariance dictates that each such interaction include a factor of the link field $U_{j+1}$, and we therefore write the corresponding interaction proportional to $f_{j+1}$.

Note that in eqn. (3.2) we have not included a Yukawa coupling corresponding to link $N+1$. Given that there is only a $U(1)$ interaction at site $N+1$, there are actually two possible such link terms (corresponding to the two Yukawa couplings of up- and down-type fermions in the standard model). We will analyze the model in the limit where the lightest fermion eigenstates (which we identify with the standard model fermions) are massless. The absence of the Yukawa couplings at site $N+1$ insures $^{\dagger}$ that the right-handed components of these massless modes are localized entirely at site $N+1$. For simplicity, in what follows we will also assume flavor universality, i.e. that the same five-dimensional fermion mass matrix applies to all flavors of fermions.

In this limit, only the left-handed components of the massless fermions are delocalized, and their behavior is characterized by a wavefuntion

$$
\left|\psi_{L}\right\rangle=\left(\begin{array}{c}
\alpha_{0} \\
\alpha_{1} \\
\vdots \\
\alpha_{N}
\end{array}\right)
$$

where the $\alpha_{j}$ are complex parameters. Denoting $\left|\alpha_{i}\right|^{2} \equiv x_{i}$ and recognizing that

$$
\sum_{i=0}^{N} x_{i}=1
$$

we find that the couplings of the ordinary (zero-mode) fermions in this model may be written

$$
\mathcal{L}_{f}=\vec{J}_{L}^{\mu} \cdot\left(\sum_{i=0}^{N} x_{i} \vec{A}_{\mu}^{i}\right)+J_{Y}^{\mu} A_{\mu}^{N+1}
$$

As usual, $\vec{J}_{L}^{\mu}$ denotes the isotriplet of left-handed weak fermion currents and $J_{Y}^{\mu}$ is the fermion hypercharge current.

The values $x_{i}$ depend on the details of the model chosen - in particular, on the form of the bulk mass, warping, and boundary conditions chosen for the fermions [39, 40]. For the first part of our discussion, we will consider the parameters $x_{i}$ as arbitrary. Later on, we will focus on a particular choice for the $x_{i}$, a so-called "ideal delocalization" which minimizes the values of corrections to precision electroweak variables.

\subsection{Re-interpreting fermion delocalization}

As noted earlier in 41, 42, the "delocalized" fermion coupling in deconstructed Higgsless models, eqn. (3.5), may also be written using the Goldstone boson fields of the Moose in

\footnotetext{
${ }^{\dagger}$ This corresponds to the limit in which the coupling $t_{R} \rightarrow 0$ in ref. 40 .
} 
fig. 1. For each $j$, the non-linear sigma model field $U_{j}$ in eqn. 2.1) corresponds to link $j$ of the moose and transforms under the adjacent $S U(2)_{j-1} \times S U(2)_{j}$ groups as $U_{j} \rightarrow V_{j-1} U_{j} V_{j}^{\dagger}$. Using these fields, define

$$
W_{k}=U_{1} \cdot U_{2} \cdots U_{k}
$$

which transforms as $W_{k} \rightarrow V_{0} W_{i} V_{k}^{\dagger}$ under $S U(2)_{0} \times S U(2)_{k}$. Consider the current operator

$$
\operatorname{Tr}\left(\frac{\sigma^{a}}{2} W_{k}^{\dagger} i D_{\mu} W_{k}\right) \rightarrow+\frac{1}{2}\left(A_{0 \mu}^{a}-A_{k \mu}^{a}\right),
$$

where the $\sigma^{a}$ are the Pauli matrices, $D_{\mu}$ is the appropriate covariant derivative, and where we have specified the form of this operator in unitary gauge in which all the link fields $U_{j} \equiv \mathcal{I}$.

In this language, the fermions' weak couplings may be written (using eqn. (3.4))

$$
\vec{J}_{L}^{\mu} \cdot\left[\vec{A}_{\mu}^{0}-\sum_{k=1}^{N} 2 x_{k} \operatorname{Tr}\left(\frac{\vec{\sigma}}{2} W_{k}^{\dagger} i D_{\mu} W_{k}\right)\right] .
$$

From this point of view, the fermions are charged only under $S U(2)_{0}$ and the apparent delocalization comes about from couplings to the Goldstone-boson fields.

Note that, in the gauge-boson normalization we are using, the linear combinations of gauge fields $A_{0 \mu}^{a}-A_{k \mu}^{a}$ are strictly orthogonal to the photon

$$
A_{\mu}^{\gamma} \propto A_{0 \mu}^{3}+A_{1 \mu}^{3}+\ldots+A_{N+1 \mu}^{3} .
$$

Hence, the couplings of eqn. (3.8) result in a modification of the $Z$ and $W$-couplings whose size depends on the $x_{k}$ and the admixture of $A_{0}-A_{k}$ in the mass-eigenstate $W$ and $Z$ fields. The couplings of eqn. (3.8) do not modify the photon coupling.

\section{Correlation Functions and Consistency Relations}

\subsection{Correlation Functions}

Recalling that fermions may be charged under any of the single $S U(2)$ gauge groups, as well as under the single $U(1)$ group at the $N+1$ site, neutral current four-fermion processes may be derived from the Lagrangian

$$
\begin{aligned}
\mathcal{L}_{n c}=- & \frac{1}{2}\left[\sum_{i, j=0}^{N} x_{i} x_{j} g_{i} g_{j} D_{i, j}^{Z}\left(Q^{2}\right)\right] J_{3}^{\mu} J_{3 \mu}-\left[\sum_{i=0}^{N} x_{i} g_{i} g_{N+1} D_{i, N+1}^{Z}\left(Q^{2}\right)\right] J_{3}^{\mu} J_{Y \mu} \\
& -\frac{1}{2}\left[g_{N+1}^{2} D_{N+1, N+1}^{Z}\left(Q^{2}\right)\right] J_{Y}^{\mu} J_{Y \mu}
\end{aligned}
$$

and charged-current process from

$$
\mathcal{L}_{c c}=-\frac{1}{2}\left[\sum_{i, j=0}^{N} x_{i} x_{j} g_{i} g_{j} D_{i, j}^{W}\left(Q^{2}\right)\right] J_{+}^{\mu} J_{-\mu} .
$$


where $D_{i, j}$ is the $(i, j)$ element of the appropriate gauge field propagator matrix. We can define correlation functions between fermion currents at given sites as

$$
\left[G_{N C}\left(Q^{2}\right)\right]_{i, j}=g_{i} g_{j} D_{i, j}^{Z}\left(Q^{2}\right), \quad\left[G_{C C}\left(Q^{2}\right)\right]_{i, j}=g_{i} g_{j} D_{i, j}^{W}\left(Q^{2}\right) .
$$

The hypercharge correlation function $\left[G_{N C}\left(Q^{2}\right)\right]_{Y Y}=\left[G_{N C}\left(Q^{2}\right)\right]_{N+1, N+1}$ depends only on the single site with a $U(1)$ gauge group. This correlation function is the same as for the simplest "Case I" model with localized fermions discussed in [24

$$
\left[G_{\mathrm{NC}}\left(Q^{2}\right)\right]_{N+1, N+1}=\left[G_{\mathrm{NC}}\left(Q^{2}\right)\right]_{Y Y}=\frac{e^{2} M_{Z}^{2}\left(Q^{2}+M_{W}^{2}\right)}{Q^{2} M_{W}^{2}\left(Q^{2}+M_{Z}^{2}\right)}\left[\prod_{w=1}^{N} \frac{Q^{2}+\mathrm{m}_{W w}^{2}}{\mathrm{~m}_{W w}^{2}}\right]\left[\prod_{z=1}^{N} \frac{\mathrm{m}_{Z z}^{2}}{Q^{2}+\mathrm{m}_{Z z}^{2}}\right]
$$

The delocalization of the fermions has no effect on this correlation function.

The full correlation function for the fermion currents $J_{3}^{\mu}$ and $J_{Y}^{\mu}$ is

$$
\left[G_{\mathrm{NC}}\left(Q^{2}\right)\right]_{W Y}=\sum_{i=0}^{N} x_{i}\left[G_{\mathrm{NC}}\left(Q^{2}\right)\right]_{i, N+1}
$$

where we have used eqn. (3.5) to include the appropriate contribution from each site to which fermions couple. By direct evaluation, following the analysis of [24], we find the relevant elements of the propagator matrix have the form

$$
\begin{aligned}
& {\left[G_{\mathrm{NC}}\left(Q^{2}\right)\right]_{0, N+1}=\frac{e^{2} M_{Z}^{2}}{Q^{2}\left(Q^{2}+M_{Z}^{2}\right)}\left[\prod_{z=1}^{N} \frac{\mathrm{m}_{Z z}^{2}}{Q^{2}+\mathrm{m}_{Z z}^{2}}\right]} \\
& {\left[G_{\mathrm{NC}}\left(Q^{2}\right)\right]_{i, N+1}=\frac{e^{2} M_{Z}^{2}}{Q^{2}\left(Q^{2}+M_{Z}^{2}\right)}\left[\prod_{\hat{i}=1}^{i}\left(1+\frac{Q^{2}}{\mathrm{~m}_{i \hat{i}}^{2}}\right)\right]\left[\prod_{z=1}^{N} \frac{\mathrm{m}_{Z z}^{2}}{Q^{2}+\mathrm{m}_{Z z}^{2}}\right] .}
\end{aligned}
$$

Finally, the full correlation functions for weak currents are

$$
\left[G_{\mathrm{NC}, \mathrm{CC}}\right]_{W W}=\sum_{i, j=0}^{N} x_{i} x_{j}\left[G_{\mathrm{NC}, \mathrm{CC}}\right]_{i, j}
$$

We discuss these correlation functions in subsection 4.3.

\subsection{Spectral Decomposition and Residue Consistency Relations}

Each correlation function may be written in a spectral decomposition in terms of the mass eigenstates and their corresponding pole residues, $\xi$, as follows:

$$
\left[G_{\mathrm{NC}}\left(Q^{2}\right)\right]_{Y Y}=\frac{\left[\xi_{\gamma}\right]_{Y Y}}{Q^{2}}+\frac{\left[\xi_{Z}\right]_{Y Y}}{Q^{2}+M_{Z}^{2}}+\sum_{z=1}^{N} \frac{\left[\xi_{Z z}\right]_{Y Y}}{Q^{2}+\mathrm{m}_{Z z}^{2}}
$$

\footnotetext{
${ }^{\ddagger}$ A Case I model is a linear moose with a set of $S U(2)$ groups adjacent to a set of $U(1)$ groups; all fermions get their hypercharge from the $U(1)$ adjacent to the $S U(2)$ groups.
} 


$$
\begin{gathered}
{\left[G_{\mathrm{NC}}\left(Q^{2}\right)\right]_{W Y}=\frac{\left[\xi_{\gamma}\right]_{W Y}}{Q^{2}}+\frac{\left[\xi_{Z}\right]_{W Y}}{Q^{2}+M_{Z}^{2}}+\sum_{z=1}^{N} \frac{\left[\xi_{Z z}\right]_{W Y}}{Q^{2}+\mathrm{m}_{Z z}^{2}},} \\
{\left[G_{\mathrm{NC}}\left(Q^{2}\right)\right]_{W W}=\frac{\left[\xi_{\gamma}\right]_{W W}}{Q^{2}}+\frac{\left[\xi_{Z}\right]_{W W}}{Q^{2}+M_{Z}^{2}}+\sum_{z=1}^{N} \frac{\left[\xi_{Z z}\right]_{W W}}{Q^{2}+\mathrm{m}_{Z z}^{2}},} \\
{\left[G_{\mathrm{CC}}\left(Q^{2}\right)\right]_{W W}=\frac{\left[\xi_{W}\right]_{W W}}{Q^{2}+M_{W}^{2}}+\sum_{w=1}^{N} \frac{\left[\xi_{W w}\right]_{W W}}{Q^{2}+\mathrm{m}_{W w}^{2}},}
\end{gathered}
$$

All poles should be simple (i.e. there should be no degenerate mass eigenvalues) because we are analyzing the discrete version of a self-adjoint operator on a finite interval. Since the neutral bosons couple to only two physically distinct currents, $J_{3}^{\mu}$ and $J_{Y}^{\mu}$, the three sets of residues in equations (4.8)-(4.9) must be related. Specifically, they satisfy the $N+1$ consistency conditions,

$$
\left[\xi_{Z}\right]_{W W}\left[\xi_{Z}\right]_{Y Y}=\left(\left[\xi_{Z}\right]_{W Y}\right)^{2}, \quad\left[\xi_{Z z}\right]_{W W}\left[\xi_{Z z}\right]_{Y Y}=\left(\left[\xi_{Z z}\right]_{W Y}\right)^{2}
$$

In the case of the photon, charge universality further implies

$$
e^{2}=\left[\xi_{\gamma}\right]_{W W}=\left[\xi_{\gamma}\right]_{W Y}=\left[\xi_{\gamma}\right]_{Y Y}
$$

Finally, we note that the residues of the poles appearing in the spectral representations of eqns. (4.8) - 4.11) are directly related to the gauge boson eigenvectors, $v_{i}^{W}$ and $v_{i}^{Z}$. To see this, recall that each residue is the product of the couplings of the related gauge boson to the appropriate fermion currents

$$
\begin{aligned}
{\left[\xi_{W}\right]_{W W} } & =g_{W}^{2}, & {\left[\xi_{Z}\right]_{W W} } & =\left(g_{Z}^{W}\right)^{2} \\
{\left[\xi_{Z}\right]_{W Y} } & =g_{Z}^{W} g_{Z}^{Y}, & {\left[\xi_{Z}\right]_{Y Y} } & =\left(g_{Z}^{Y}\right)^{2}
\end{aligned}
$$

(which also leads back to the consistency relations in (4.12) above). The coupling of a gauge boson mass eigenstate to a fermion current is the sum of the contributions from each site

$$
\begin{aligned}
g_{W} & =\sum_{j=0}^{N} x_{j} g_{j} v_{j}^{W}, \\
g_{Z}^{W} & =\sum_{j=0}^{N} x_{j} g_{j} v_{j}^{Z}, \\
g_{Z}^{Y} & =g_{N+1} v_{N+1}^{Z},
\end{aligned}
$$

and therefore reflects the fermion and gauge boson wave-functions and the site-dependent couplings. So the residues are indeed related to the eigenvectors. 


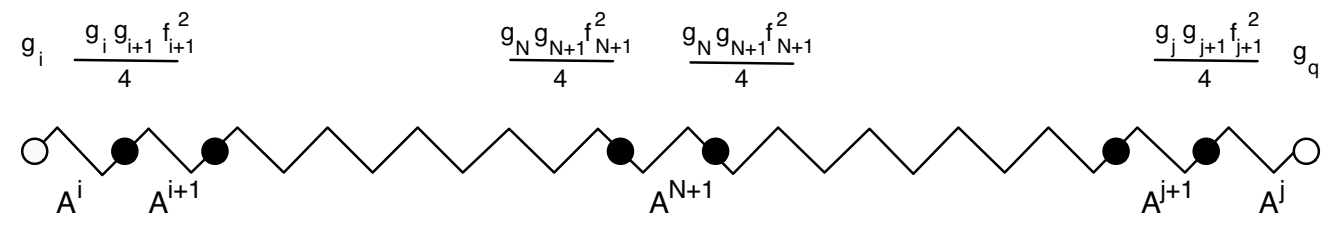

Figure 2: Leading diagram at high- $Q^{2}\left(Q^{2} \gg \mathrm{m}_{Z z, W w}^{2}\right.$ for $\left.w, z>0\right)$ which distinguishes $\left[G_{\mathrm{NC}}\left(Q^{2}\right)\right]_{i, j}$ from $\left[G_{\mathrm{CC}}\left(Q^{2}\right)\right]_{i, j}$.

\subsection{Consistency Relations Among Correlation Functions}

Consider the difference of charged and neutral current correlation functions

$$
\left[G_{\mathrm{NC}}\left(Q^{2}\right)\right]_{i, j}-\left[G_{\mathrm{CC}}\left(Q^{2}\right)\right]_{i, j},
$$

where, without loss of generality, we may take $i \leq j$. At high- $Q^{2}$, the leading contribution to the difference is shown in fig. 2, and therefore at large momenta

$$
\left[G_{\mathrm{NC}}\left(Q^{2}\right)\right]_{i, j}-\left[G_{\mathrm{CC}}\left(Q^{2}\right)\right]_{i, j} \propto \frac{g_{N+1}^{2}}{\left(Q^{2}\right)^{2 N-i-j+3}} .
$$

The only consistent way to achieve the high energy behavior while including the poles appropriate for the charged and neutral correlation functions is to write

$$
\left[G_{\mathrm{NC}}\left(Q^{2}\right)\right]_{i, j}-\left[G_{\mathrm{CC}}\left(Q^{2}\right)\right]_{i, j}=\frac{e^{2} M_{W}^{2} M_{Z}^{2} \mathcal{R}\left(Q^{2}\right)}{Q^{2}\left[Q^{2}+M_{W}^{2}\right]\left[Q^{2}+M_{Z}^{2}\right]}\left[\prod_{w=1}^{N} \frac{\mathrm{m}_{W w}^{2}}{Q^{2}+\mathrm{m}_{W w}^{2}}\right]\left[\prod_{z=1}^{N} \frac{\mathrm{m}_{Z z}^{2}}{Q^{2}+\mathrm{m}_{Z z}^{2}}\right],
$$

where the function $\mathcal{R}\left(Q^{2}\right)$ is a polynomial in $Q^{2}$ of order $i+j$ and $\mathcal{R}(0)=1$ in order to satisfy charge universality. Furthermore, by direct evaluation [24], we see that

$$
\left[G_{\mathrm{NC}, \mathrm{CC}}\left(Q^{2}\right)\right]_{i, j} \propto\left[\prod_{\hat{i}=1}^{i}\left(1+\frac{Q^{2}}{\mathrm{~m}_{i \hat{i}}^{2}}\right)\right]
$$

and therefore the polynomial satisfies the $i$ conditions $\mathcal{R}\left(-\mathrm{m}_{i \hat{i}}^{2}\right)=0$.

Recall the correlation functions $\left[G_{\mathrm{NC}}\left(Q^{2}\right)\right]_{i, N+1}$ and $\left[G_{\mathrm{NC}}\left(Q^{2}\right)\right]_{j, N+1}$, which can be written as in eqn. (4.6), and the correlation function $\left[G_{\mathrm{NC}}\left(Q^{2}\right)\right]_{N+1, N+1} \equiv\left[G_{\mathrm{NC}}\left(Q^{2}\right)\right]_{Y Y}$. We may expand each of these, as well as $\left[G_{\mathrm{NC}}\left(Q^{2}\right)\right] i, j$ in a spectral representation like the one employed in eqns. (4.8 - 4.11). The residues of these correlation functions will therefore satisfy the $N+1$ conditions

$$
\left[\xi_{Z}\right]_{i, j}=\frac{\left[\xi_{Z}\right]_{i, N+1}\left[\xi_{Z}\right]_{j, N+1}}{\left[\xi_{Z}\right]_{N+1, N+1}}, \quad\left[\xi_{Z z}\right]_{i, j}=\frac{\left[\xi_{Z z}\right]_{i, N+1}\left[\xi_{Z z}\right]_{j, N+1}}{\left[\xi_{Z z}\right]_{N+1, N+1}}
$$

The combination of these $N+1$ conditions and the $i$ conditions $\mathcal{R}\left(-\mathrm{m}_{i \hat{i}}^{2}\right)=0$ overdetermines $\underline{\mathcal{R}}\left(Q^{2}\right)$, and we find a consistency condition among correlation functions ${ }^{\S}$

\footnotetext{
${ }^{\S}$ This expression can also be derived from the consistency relation given in 24 for $\left[G_{\mathrm{NC}}\left(Q^{2}\right)\right]_{j, j}-$ $\left[G_{\mathrm{CC}}\left(Q^{2}\right)\right]_{j, j}$, and using the explicit forms of the correlation functions.
} 


$$
\left[G_{\mathrm{NC}}\left(Q^{2}\right)\right]_{i, j}-\left[G_{\mathrm{CC}}\left(Q^{2}\right)\right]_{i, j}=\frac{\left[G_{\mathrm{NC}}\left(Q^{2}\right)\right]_{i, N+1}\left[G_{\mathrm{NC}}\left(Q^{2}\right)\right]_{j, N+1}}{\left[G_{\mathrm{NC}}\left(Q^{2}\right)\right]_{N+1, N+1}} .
$$

Direct evaluation of the correlation functions on the right hand side of this expression (using eqns. (4.4) and (4.6)) yields 9

$$
\begin{aligned}
& {\left[G_{\mathrm{NC}}\left(Q^{2}\right)\right]_{i, j}-\left[G_{\mathrm{CC}}\left(Q^{2}\right)\right]_{i, j}=\frac{e^{2} M_{Z}^{2} M_{W}^{2}}{Q^{2}\left(Q^{2}+M_{Z}^{2}\right)\left(Q^{2}+M_{W}^{2}\right)} \times} \\
& {\left[\prod_{z=1}^{N} \frac{\mathrm{m}_{Z_{z}}^{2}}{Q^{2}+\mathrm{m}_{Z_{z}}^{2}}\right]\left[\prod_{w=1}^{N} \frac{\mathrm{m}_{W_{w}}^{2}}{Q^{2}+\mathrm{m}_{W_{w}}^{2}}\right]\left[\prod_{\hat{i}=1}^{i}\left(1+\frac{Q^{2}}{\mathrm{~m}_{i \hat{i}}^{2}}\right)\right]\left[\prod_{\hat{j}=1}^{j}\left(1+\frac{Q^{2}}{\mathrm{~m}_{j \hat{j}}^{2}}\right)\right]}
\end{aligned}
$$

Finally, applying eqn. (4.7) to the LHS of 4.25) and eqns. (4.5) and (4.4) to its RHS reveals that the full correlation functions satisfy the same consistency relations

$$
\left[G_{\mathrm{NC}}\left(Q^{2}\right)\right]_{W W}-\left[G_{\mathrm{CC}}\left(Q^{2}\right)\right]_{W W}=\frac{\left(\left[G_{\mathrm{NC}}\left(Q^{2}\right)\right]_{W Y}\right)^{2}}{\left[G_{\mathrm{NC}}\left(Q^{2}\right)\right]_{Y Y}}
$$

as those [24] in Case I models with fermions localized at a single site.

\section{$4.4 W$ Eigenvector}

Since the residues of the poles of the correlation functions are related to the gauge couplings $\left(g_{i}\right)$ and eigenvectors $\left(v_{i}\right)$, as in Section 4.2, we may use our expressions for the correlation functions to find the components of the massive eigenvectors in terms of the spectrum and gauge couplings. Here, we derive the form of the $W$ eigenvector from the consistency condition of eqn. (4.25) and the relation

$$
\left[\xi_{W}\right]_{i, j}=g_{i} g_{j} v_{i}^{W} v_{j}^{W},
$$

Computing the residue of the $W$ pole in (4.25) and expanding it in inverse powers of the higher KK masses we find

$$
\left[\xi_{W}\right]_{i, j}=\left(\frac{e^{2}}{1-\frac{M_{W}^{2}}{M_{Z}^{2}}}\right)\left[1+M_{W}^{2}\left(\Sigma_{W}+\Sigma_{Z}\right)\right]\left[\prod_{\hat{i}=1}^{i}\left(1-\frac{M_{W}^{2}}{\mathrm{~m}_{i \hat{i}}^{2}}\right)\right]\left[\prod_{\hat{j}=1}^{j}\left(1-\frac{M_{W}^{2}}{\mathrm{~m}_{j \hat{j}}^{2}}\right)\right]
$$

and hence, using (4.27),

$$
g_{i} v_{i}^{W}=\frac{e}{\sqrt{1-\frac{M_{W}^{2}}{M_{Z}^{2}}}}\left[1+\frac{M_{W}^{2}}{2}\left(\Sigma_{W}+\Sigma_{Z}\right)\right]\left[\prod_{\hat{i}=1}^{i}\left(1-\frac{M_{W}^{2}}{\mathrm{~m}_{i \hat{i}}^{2}}\right)\right] .
$$

Note that $v_{N+1}^{W} \equiv 0$, as it must be given the form of the model in fig. 11. Maintaining positive values for the remaining elements of the $v^{W}$ eigenvector (corresponding to a nodeless wavefunction in the continuum limit) implies that $M_{W}^{2}<\mathrm{m}_{i \hat{i}}^{2}$ for all $i$ and $\hat{i}$.

\footnotetext{
${ }^{\mathbb{I}}$ Here $\mathrm{m}_{j \hat{j}}^{2}$ are the eigenvalues of $M_{[0, j)}^{2}$.
} 
For $i$ large, close to $N+1$, we expect that the matrix $M_{[0, i)}^{2}$ will have a light eigenvalue close to $M_{W}^{2}$. For $i$ small, however, we expect that we may expand the product in powers of $M_{W}^{2} / \mathrm{m}_{i \hat{i}}^{2}$ and find the approximate form

$$
g_{i} v_{i}^{W}=\frac{e}{\sqrt{1-\frac{M_{W}^{2}}{M_{Z}^{2}}}}\left[1+\frac{M_{W}^{2}}{2}\left(\Sigma_{W}+\Sigma_{Z}\right)-M_{W}^{2} \Sigma_{[0, i)}\right] .
$$

For small $i$ therefore, to leading order in the inverse mass-squared expansion, $g_{i} v_{i}^{W}$ is approximately a constant.

\section{Ideal Delocalization}

A big question remains: what kinds of delocalization schemes produce viable models? Viability depends, in part, on satisfying constraints from precision electroweak corrections, so we remind the reader of the relevant definitions. Then we introduce "ideal delocalization" which guarantees that the corrections can be made small. We discuss this in deconstructed language. Then in section 6 , we show that the results hold neatly in the continuum.

\subsection{Electroweak Parameters}

As we have shown in [22], the most general amplitude for low-energy four-fermion neutral weak current processes in any "universal" model [15] may be written as"

$$
\begin{aligned}
-\mathcal{M}_{N C}=e^{2} \frac{\mathcal{Q} \mathcal{Q}^{\prime}}{Q^{2}} & +\frac{\left(I_{3}-s^{2} \mathcal{Q}\right)\left(I_{3}^{\prime}-s^{2} \mathcal{Q}^{\prime}\right)}{\left(\frac{s^{2} c^{2}}{e^{2}}-\frac{S}{16 \pi}\right) Q^{2}+\frac{1}{4 \sqrt{2} G_{F}}\left(1-\alpha T+\frac{\alpha \delta}{4 s^{2} c^{2}}\right)} \\
& +\sqrt{2} G_{F} \frac{\alpha \delta}{s^{2} c^{2}} I_{3} I_{3}^{\prime}+4 \sqrt{2} G_{F}(\Delta \rho-\alpha T)\left(\mathcal{Q}-I_{3}\right)\left(\mathcal{Q}^{\prime}-I_{3}^{\prime}\right)
\end{aligned}
$$

and the matrix element for charged current process may be written

$$
-\mathcal{M}_{\mathrm{CC}}=\frac{\left(I_{+} I_{-}^{\prime}+I_{-} I_{+}^{\prime}\right) / 2}{\left(\frac{s^{2}}{e^{2}}-\frac{S}{16 \pi}\right) Q^{2}+\frac{1}{4 \sqrt{2} G_{F}}\left(1+\frac{\alpha \delta}{4 s^{2} c^{2}}\right)}+\sqrt{2} G_{F} \frac{\alpha \delta}{s^{2} c^{2}} \frac{\left(I_{+} I_{-}^{\prime}+I_{-} I_{+}^{\prime}\right)}{2} .
$$

The parameter $s^{2}$ is defined implicitly in these expressions as the ratio of the $\mathcal{Q}$ and $I_{3}$ couplings of the $Z$ boson. $\Delta \rho$ corresponds to the deviation from unity of the ratio of the strengths of low-energy isotriplet weak neutral-current scattering and charged-current scattering. $S$ and $T$ are the familiar oblique electroweak parameters [27, 28, 29], as determined by examining the on-shell properties of the $Z$ and $W$ bosons. The contact interactions proportional to $\alpha \delta$ and $(\Delta \rho-\alpha T)$ correspond to "universal non-oblique" corrections arising from the exchange of heavy KK modes. Finally, the consistency relation, eqn. (4.26), insures that $\Delta \rho=0$ in any Case I model [20], regardless of fermion delocalization.

\footnotetext{
"See 22] for a discussion of the correspondence between the "on-shell" parameters defined here, and the zero-momentum parameters defined in 15]. Note that $U$ is shown in 22 to be zero to the order we consider in this paper.
} 


\subsection{Optimizing the Fermion Wavefunction: $\alpha \delta$}

We now consider how to choose the form of the delocalized fermion wavefunction so as to minimize the deviations in the electroweak parameters. Consider first the parameter $\alpha \delta-$ from the form of eqn. (5.2) we see that this deviation arises from the exchange of massive KK modes. Therefore,

$$
\alpha \delta \propto \sum_{w=1}^{N} \sum_{i, j} x_{i} x_{j} \frac{\left[\xi_{W_{w}}\right] i, j}{\mathrm{~m}_{W_{w}}^{2}}
$$

where $\left[\xi_{W_{w}}\right]_{i, j}$ is the pole residue of $\left[G_{\mathrm{CC}}\left(Q^{2}\right)\right]_{i, j}$ at $Q^{2}=\mathrm{m}_{W_{w}}^{2}$. In analogy to eqn. (4.27), the pole residues are related to the $W_{w}$ wave functions $v_{i}^{W_{w}}$,

$$
\left[\xi_{W_{w}}\right]_{i, j}=g_{i} g_{j} v_{i}^{W_{w}} v_{j}^{W_{w}} .
$$

For a given fermion wavefunction $x_{i}$, we thus find

$$
\alpha \delta \propto \sum_{w=1}^{N} \frac{\left(\sum_{i} g_{i} x_{i} v_{i}^{W_{w}}\right)^{2}}{\mathrm{~m}_{W_{w}}^{2}},
$$

and $\alpha \delta$ is therefore a positive-semi-definite parameter.

To minimize $\alpha \delta$, we exploit the fact that the eigenvector for the massive $W$ and those for each of the KK modes are mutually orthogonal

$$
\sum_{i} v_{i}^{W} v_{i}^{W_{w}}=0
$$

Since $\alpha \delta$ involves the product $x_{i} v_{i}^{W_{w}}$, we choose our "ideally delocalized" fermion wavefunction $x_{i}$ to be related to the form of the $W$ wavefunction

$$
g_{i} x_{i}=\mathcal{N} v_{i}^{W},
$$

where the normalization factor $\mathcal{N}$ is fixed by the constraint $\sum_{i} x_{i}=1$. By construction, the contact interaction $\alpha \delta$ vanishes for an ideally delocalized fermion. As noted earlier, we may choose the lightest gauge boson eigenvector to be positive definite (corresponding to a nodeless wavefunction in the continuum limit), and therefore $x_{i} \geq 0$ for ideal delocalization.

\section{$5.3 \alpha S$ and $\alpha T$ for an Ideally Delocalized Fermion}

Consider the following combination of correlation functions with the fermion wavefunction

$$
\sum_{i} x_{i}\left(\left[G_{\mathrm{NC}}\left(Q^{2}\right)\right]_{i, j}-\left[G_{\mathrm{CC}}\left(Q^{2}\right)\right]_{i, j}\right) .
$$

If we assume an ideally delocalized fermion, as defined above, it cannot couple to the higher

charged KK modes. Therefore the combination of correlation functions in eqn. (5.8) cannot have poles at $Q^{2}=-\mathrm{m}_{W w}^{2}$. From eqn. (4.25), we thus find

$$
\sum_{i=0}^{N} x_{i}\left[\prod_{\hat{i}=1}^{i}\left(1+\frac{Q^{2}}{\mathrm{~m}_{i \hat{i}}^{2}}\right)\right]=\mathcal{A}\left(Q^{2}\right)\left[\prod_{w=1}^{N} \frac{Q^{2}+\mathrm{m}_{W_{w}}^{2}}{\mathrm{~m}_{W_{w}}^{2}}\right]
$$


for some polynomial $\mathcal{A}\left(Q^{2}\right)$. The left hand side of eqn. (5.9), however, is at most a polynomial of degree $N$ in $Q^{2}$; therefore $\mathcal{A}\left(Q^{2}\right)$ must be a constant, which we denote $\mathcal{A}$.

Applying eqn. (5.9), we may now evaluate $\left[G_{\mathrm{NC}}\left(Q^{2}\right)\right]_{W Y}$ from eqns. (4.5) and (4.6), and $\left[G_{\mathrm{NC}}\left(Q^{2}\right)\right]_{W W}-\left[G_{\mathrm{CC}}\left(Q^{2}\right)\right]_{W W}$ from eqns. (4.7) and (4.26), to find

$$
\left[G_{\mathrm{NC}}\left(Q^{2}\right)\right]_{W Y}=\frac{\mathcal{A} e^{2} M_{Z}^{2}}{Q^{2}\left(Q^{2}+M_{Z}^{2}\right)}\left[\prod_{z=1}^{N} \frac{\mathrm{m}_{Z_{z}}^{2}}{Q^{2}+\mathrm{m}_{Z_{z}}^{2}}\right]\left[\prod_{w=1}^{N} \frac{Q^{2}+\mathrm{m}_{W_{w}}^{2}}{\mathrm{~m}_{W_{w}}^{2}}\right],
$$

and

$$
\begin{aligned}
& {\left[G_{\mathrm{NC}}\left(Q^{2}\right)\right]_{W W}-\left[G_{\mathrm{CC}}\left(Q^{2}\right)\right]_{W W}=} \\
& \frac{\mathcal{A}^{2} e^{2} M_{Z}^{2} M_{W}^{2}}{Q^{2}\left(Q^{2}+M_{Z}^{2}\right)\left(Q^{2}+M_{W}^{2}\right)}\left[\prod_{\hat{z}=1}^{N} \frac{\mathrm{m}_{Z_{\hat{z}}}^{2}}{Q^{2}+\mathrm{m}_{Z_{\hat{z}}}^{2}}\right]\left[\prod_{w=1}^{N} \frac{Q^{2}+\mathrm{m}_{W_{w}}^{2}}{\mathrm{~m}_{W_{w}}^{2}}\right] .
\end{aligned}
$$

Note that these expressions are specific to the case of an ideally delocalized fermion. The normalization $\mathcal{A}$ can be determined by examining the residue of the photon pole in either eqn. (5.10) or (5.11). From $\left[G_{\mathrm{NC}}\left(Q^{2}\right)\right]_{W Y}$, for example, we have

$$
\left[\xi_{\gamma}\right]_{W Y}=\mathcal{A} e^{2}=e^{2} \quad \Rightarrow \quad \mathcal{A}=1
$$

by charge universality, eqn. (4.13).

We have previously shown [24, 20] that $\alpha S$ may be computed by examining the residue of the correlation function $\left[G_{\mathrm{NC}}\left(Q^{2}\right)\right]_{W Y}$ at $Q^{2}=-M_{Z}^{2}$

$$
\left[\xi_{Z}\right]_{W Y}=-e^{2}\left[1+\frac{\alpha S}{4 s^{2} c^{2}}\right] .
$$

Evaluating the residue from eqn. (5.10), and expanding in inverse powers of the KK masses, we find

$$
\left[\xi_{Z}\right]_{W Y}=-e^{2}\left[1+M_{Z}^{2}\left(\Sigma_{Z}-\Sigma_{W}\right)\right]
$$

and therefore conclude

$$
\alpha S=4 s^{2} c^{2} M_{Z}^{2}\left(\Sigma_{Z}-\Sigma_{W}\right) .
$$

The parameter $\alpha T$ is independent of fermion delocalization, and is computed in [24, 20] to be

$$
\alpha T=s^{2} M_{Z}^{2}\left(\Sigma_{Z}-\Sigma_{W}\right)
$$

In the continuum Higgsless models in warped and flat space discussed in section 6 [3, 17], we find [43] that $\alpha S$ and $\alpha T$ are small and slightly negative .

It is now straightforward to calculate the electroweak parameters of Barbieri et al. [15, 22],

$$
\begin{aligned}
\hat{S} & =\frac{1}{4 s^{2}}\left(\alpha S+4 c^{2}(\Delta \rho-\alpha T)+\frac{\alpha \delta}{c^{2}}\right) \\
\hat{T} & =\Delta \rho \\
W & =\frac{\alpha \delta}{4 s^{2} c^{2}} \\
Y & =\frac{c^{2}}{s^{2}}(\Delta \rho-\alpha T)
\end{aligned}
$$


and for ideally delocalized fermions we obtain

$$
\hat{S}=\hat{T}=W=0, \quad Y=M_{W}^{2}\left(\Sigma_{W}-\Sigma_{Z}\right) .
$$

\subsection{Normalization of Distribution of Ideally Delocalized Fermion}

The normalization $\mathcal{N}$ of the ideally delocalized fermion in eqn. (5.7) can be determined from the $W$-pole residue $\left[\xi_{W}\right]_{W W}$ as computed from eqn. (5.11). Expanding the residue in inverse powers of the KK masses, we find

$$
\left[\xi_{W}\right]_{W W}=\left(\sum_{i} x_{i} g_{i} v_{i}^{W}\right)^{2}=\frac{e^{2}}{\left(1-\frac{M_{W}^{2}}{M_{Z}^{2}}\right)}\left[1+M_{W}^{2}\left(\Sigma_{Z}-\Sigma_{W}\right)\right]
$$

and we recall that $\left[\xi_{W}\right]_{W W}=g_{W}^{2}$ from eqn. (4.14). Taking the dot product of eqn. 5.7 with $v_{i}^{W}$, we see that the normalization $\mathcal{N}$ is equal to the $W$ coupling to fermions

$$
\mathcal{N}=\mathcal{N} \sum_{i} v_{i}^{W} v_{i}^{W}=\sum_{i} x_{i} g_{i} v_{i}^{W}=g_{W}
$$

Therefore

$$
\mathcal{N}=\frac{e}{\sqrt{1-\frac{M_{W}^{2}}{M_{Z}^{2}}}}\left[1+\frac{M_{W}^{2}}{2}\left(\Sigma_{Z}-\Sigma_{W}\right)\right]
$$

and the profile of the ideally delocalized fermion is related to the $W$ wavefunction by

$$
g_{i} x_{i}=g_{W} v_{i}^{W}
$$

\section{Two Examples in the Continuum}

We briefly discuss ideal fermion delocalization in two continuum Higgsless models. In this paper, we will describe the correspondence between the $S U(2)$ sectors of the deconstructed and continuum models, and display the results for the $W$ wavefunction and ideal fermion delocalization; details of the calculations and a discussion of the $U(1)$ sector of these models will be presented in 43 .

\subsection{Warped Higgsless Models}

We first consider Higgsless models in Anti-deSitter space, as described in 39. A deconstructed moose which yields this model in conformally flat coordinates has the following parameters 43]

$$
\begin{aligned}
& f_{i}^{2}=v^{2}(N+1) \\
& \frac{1}{g_{i}^{2}}=\frac{1}{b g^{2}} \log \left[\frac{i+1+(N-i) e^{-b}}{i+(N+1-i) e^{-b}}\right], \quad i=0, \ldots, N
\end{aligned}
$$


The continuum limit is taken by sending $N \rightarrow \infty$ while holding $g, v$, and $b$ fixed. The conformally flat coordinates in the fifth dimension, $z$, corresponding to the deconstructed lattice points are

$$
z_{i}=R^{\prime}\left[e^{-b}+\frac{i}{N+1}\left(1-e^{-b}\right)\right]^{1 / 2}
$$

and therefore in the continuum limit

$$
2 z_{i} \Delta z_{i}=\frac{R^{\prime 2}}{N+1}\left(1-e^{-b}\right) \rightarrow 2 z d z
$$

For $i=0,1, \ldots, N+1$, we see that

$$
R \equiv R^{\prime} e^{-b / 2} \leq z \leq R^{\prime}
$$

and hence, following [39], we identify $R \simeq 1 / M_{p l}$ with the position of the "Planck" brane and $R^{\prime} \simeq 1 / \mathrm{TeV}$ with the position of the "TeV" brane. We therefore expect

$$
b=2 \log \frac{R^{\prime}}{R} \simeq \mathcal{O}(60) .
$$

From eqns. (6.2), (6.3), and (6.4), we find that in the continuum limit the coupling becomes position-dependent

$$
\begin{aligned}
\frac{1}{g_{i}^{2}} & =\frac{1}{b g^{2}} \log \left[1+\frac{R^{\prime 2}}{N+1}\left(\frac{1-e^{-b}}{z_{i}^{2}}\right)\right], \\
& \rightarrow \frac{d z}{g_{5}^{2}(z)}=\frac{2 d z}{b g^{2} z}
\end{aligned}
$$

Note that in eqn. (6.8), we have interpreted the conformal factor in the metric used to describe the model in [39] as a position-dependent five-dimensional gauge coupling $g_{5}^{2}(z)$.

Requiring that the continuum limit of the nonlinear sigma model terms in eqn. (2.1) yield the $A_{5}$-dependent kinetic terms for a five-dimensional Yang-Mills theory fixes the correspondence between the Goldstone bosons and $A_{5}$, and implies that [25, 26]

$$
\Delta z_{i}=\frac{2}{g_{i} f_{i}} .
$$

Using

$$
\sum_{i} \Delta z_{i}=R^{\prime}-R \approx R^{\prime}
$$

we then compute the relation

$$
R^{\prime}=\frac{4}{\sqrt{b} g v}
$$

Solving the resulting continuum eigenvalue equation for the $W$ 39, 43, we find ${ }^{* *}$

$$
M_{W}^{2} \approx \frac{g^{2} v^{2}}{4}=\frac{2}{R^{\prime 2} \log \frac{R^{\prime}}{R}}
$$

\footnotetext{
${ }^{* *}$ Our result for $M_{W}^{2}$ agrees with [39] in the limit where the coupling $g_{R} \rightarrow 0$.
} 
and that near the Planck brane the $W$ wavefunction satisfies ${ }^{\dagger \dagger}$

$$
g_{i} v_{i}^{W} \rightarrow g_{5}(z) \chi^{W}(z) \simeq \text { constant },
$$

as expected from our discussion of the general properties of the $W$ eigenvector in section 4.4 .

Finally, we may compute the ideally delocalized fermion wavefunction appropriate for this model. In the continuum limit the fermion probabilities $x_{i}$ are related to the corresponding continuum wavefunction, $x_{i} \rightarrow|\psi(z)|^{2} d z$, and hence near the Planck brane we find

$$
\begin{aligned}
x_{i} & =\frac{g_{W} v_{i}^{W}}{g_{i}}=\frac{g_{W}\left(g_{i} v_{i}^{W}\right)}{g_{i}^{2}}, \\
& \rightarrow|\psi(z)|^{2} d z \propto \frac{2 d z}{b z} .
\end{aligned}
$$

The ideally delocalized fermion wavefunction therefore corresponds near the Planck brane to the "flat" wavefunction described in [39]. Since the flat and ideal wavefunctions coincide near the Planck brane where the $W$ wavefunction is concentrated, the flat wavefunction results in $\hat{S}=\hat{T}=W=0$, and therefore $\alpha S=0$ [39], up to order $1 / b^{2}$ [43].

\subsection{Flat Higgsless Models}

Next we consider delocalized fermions in a flat background spacetime, as discussed in 440]. The deconstructed version of this model has the same $f_{i}$ shown in eqn. (6.1), but rather different gauge-couplings

$$
\frac{1}{g_{i}^{2}}=\left\{\begin{array}{l}
\frac{1}{g_{0}^{2}}, \quad i=0, \\
\frac{1}{N \tilde{g}^{2}}, \quad i=1,2, \ldots, N .
\end{array}\right.
$$

The continuum limit is taken holding $g_{0}, \tilde{g}$, and $v$ fixed, and the hierarchy of masses between the $W$ and the KK modes is enforced by taking $g_{0}^{2} \ll \tilde{g}^{2}$. The fifth coordinates, $\tilde{x}$, corresponding to the deconstructed lattice points are

$$
\tilde{x}_{i}=\frac{i \pi R}{N+1},
$$

and, therefore, in the continuum limit

$$
\Delta \tilde{x}_{i}=\frac{\pi R}{N+1} \rightarrow d \tilde{x} .
$$

For $i=0,1, \ldots, N+1$, we see that

$$
0 \leq \tilde{x} \leq \pi R .
$$

The continuum limit of the gauge-couplings is then

$$
\frac{1}{g_{i}^{2}} \rightarrow\left(\frac{1}{\pi R \tilde{g}^{2}}+\frac{1}{g_{0}^{2}} \delta(\tilde{x})\right) d \tilde{x}
$$

\footnotetext{
${ }^{\dagger \dagger}$ Given the normalization of the gauge kinetic-energy terms in eqn. 2.1., it is the combination $g_{i} v_{i}^{W}$ that has a well-defined continuum limit 43].
} 
and the Lagrangian of eqn. (2.1) yields a five-dimensional Yang-Mills theory with coupling

$$
g_{5}=\tilde{g} \sqrt{\pi R},
$$

and the necessary "brane" kinetic energy terms [17].

As in the previous example, requiring that the continuum limit of the nonlinear sigma model terms in eqn. (2.1) yield the $A_{5}$-dependent kinetic energy terms for a five-dimensional Yang-Mills theory fixes the correspondence between the Goldstone bosons and $A_{5}$, and implies that 25, 26]

$$
\Delta \tilde{x}_{i}=\frac{2}{g_{i} f_{i}} .
$$

We therefore find the continuum limit

$$
\sum_{i} \Delta \tilde{x}_{i}=\pi R \rightarrow \frac{2}{\tilde{g} v}
$$

The $W$ wavefunction follows from the continuum eigenvalue equations [17, 43, and we find

$$
M_{W}^{2} \approx \frac{g_{0}^{2} v^{2}}{4}
$$

and

$$
g_{i} v_{i}^{W} \rightarrow g_{5} \chi^{W}(\tilde{x})=g_{0}\left[\left(1-\frac{\tilde{x}}{\pi R}\right)-\left(\frac{g_{0}^{2}}{6 \tilde{g}^{2}}\right)\left(1-\frac{\tilde{x}}{\pi R}\right)^{3}+\ldots\right] .
$$

The ideally delocalized fermion wavefunction in this model is then computed to be

$$
x_{i} \rightarrow|\psi(\tilde{x})|^{2} d \tilde{x} \propto\left(\frac{1}{\pi R \tilde{g}^{2}}+\frac{1}{g_{0}^{2}} \delta(\tilde{x})\right) g_{5} \chi^{W}(\tilde{x}) d \tilde{x}
$$

which, after normalization, yields

$$
|\psi(\tilde{x})|^{2} d \tilde{x}=\left[\left(1-\frac{g_{0}^{2}}{2 \tilde{g}^{2}}\right) \delta(\tilde{x})+\frac{g_{0}^{2}}{\pi R \tilde{g}^{2}}\left(1-\frac{\tilde{x}}{\pi R}\right)+\ldots\right] d \tilde{x}
$$

It may be verified that $\hat{S}=\hat{T}=W=0$ in this case; details will be given in 43.

Finally, we note that the slightly modified fermion distribution

$$
|\phi(y)|^{2} d y=\left[\left(1-\frac{B g_{0}^{2}}{2 \tilde{g}^{2}}\right) \delta(\tilde{x})+\frac{B g_{0}^{2}}{\pi R \tilde{g}^{2}}\left(1-\frac{\tilde{x}}{\pi R}\right)+\ldots\right] d \tilde{x},
$$

results [43] in a potentially nonzero value of $\alpha S$

$$
\frac{\alpha S}{4 s^{2} c^{2}}=\frac{(1-B)}{6}\left[\frac{g_{0}^{2}}{\tilde{g}^{2}}+\frac{g_{N+1}^{2}}{\tilde{g}^{2}}\right]
$$

In fact, this adjustable tree-level value of $\alpha S$ might serve to cancel contributions arising from higher-order effects 21. 


\section{Conclusions}

We have examined the properties of deconstructed Higgsless models for the case of a fermion whose $S U(2)$ properties arise from delocalization over many sites of the deconstructed lattice. We have derived expressions for the correlation functions and used these to establish a generalized consistency relation among them. We have discussed the form of the $W$ boson wavefunction and have shown that if the probability distribution of the delocalized fermions is appropriately related to the $W$ wavefunction, the precision electroweak parameters [15] $\hat{S}$, $\hat{T}$, and $W$ are exactly zero at tree-level.

Our results may be applied to any Higgsless linear moose model with multiple $S U(2)$ groups, including a large class of extended electroweak gauge theories with only a few extra vector bosons. We have briefly discussed the ideal fermion delocalization in two continuum Higgsless models, one in Anti-deSitter space and one in flat space. The details of deconstructing the continuum flat and Anti-deSitter space Higgsless models, and of computing the ideal fermion delocalization distributions and a discussion of the $U(1)$ sectors of these models, will be presented in 443.

\section{Acknowledgments}

R.S.C. and E.H.S. are supported in part by the US National Science Foundation under award PHY-0354226, and gratefully acknowledge the hospitality of the Tohoku University Theory group. M.K. is supported by a MEXT Grant-in-Aid for Scientific Research No. 14046201. M.T.'s work is supported in part by the JSPS Grant-in-Aid for Scientific Research No.16540226. H.J.H. is supported by the US Department of Energy grant DE-FG0393ER40757.

\section{References}

[1] C. Csaki, C. Grojean, H. Murayama, L. Pilo and J. Terning, Gauge theories on an interval: Unitarity without a Higgs, Phys. Rev. D 69, 055006 (2004) [arXiv:hep-ph/0305237].

[2] K. Agashe, A. Delgado, M. J. May and R. Sundrum, RS1, custodial isospin and precision tests, JHEP 0308, 050 (2003) [arXiv:hep-ph/0308036].

[3] C. Csaki, C. Grojean, L. Pilo, and J. Terning, Towards a realistic model of higgsless electroweak symmetry breaking, Phys. Rev. Lett. 92 (2004) 101802, hep-ph/0308038].

[4] R. Sekhar Chivukula, D. A. Dicus, and H.-J. He, Unitarity of compactified five dimensional yang-mills theory, Phys. Lett. B525 (2002) 175-182, hep-ph/0111016.

[5] R. S. Chivukula and H.-J. He, Unitarity of deconstructed five-dimensional yang-mills theory, Phys. Lett. B532 (2002) 121-128, [hep-ph/0201164.

[6] R. S. Chivukula, D. A. Dicus, H.-J. He, and S. Nandi, Unitarity of the higher dimensional standard model, Phys. Lett. B562 (2003) 109-117, hep-ph/0302263.

[7] H.-J. He, Higgsless deconstruction without boundary condition, arXiv:hep-ph/0412113. 
[8] P. W. Higgs, Broken symmetries, massless particles and gauge fields, Phys. Lett. 12 (1964) $132-133$.

[9] G. Cacciapaglia, C. Csaki, C. Grojean and J. Terning, Oblique corrections from Higgsless models in warped space, Phys. Rev. D 70, 075014 (2004) [arXiv:hep-ph/0401160].

[10] Y. Nomura, Higgsless theory of electroweak symmetry breaking from warped space, JHEP 11 (2003) 050, hep-ph/0309189.

[11] R. Barbieri, A. Pomarol and R. Rattazzi, Weakly coupled Higgsless theories and precision electroweak tests, Phys. Lett. B 591 (2004) 141 [arXiv:hep-ph/0310285].

[12] H. Davoudiasl, J. L. Hewett, B. Lillie and T. G. Rizzo, Higgsless electroweak symmetry breaking in warped backgrounds: constraints and signatures, Phys. Rev. D 70 (2004) 015006 [arXiv:hep-ph/0312193].

[13] G. Burdman and Y. Nomura, Holographic theories of electroweak symmetry breaking without a Higgs boson, Phys. Rev. D 69 (2004) 115013 [arXiv:hep-ph/0312247].

[14] H. Davoudiasl, J. L. Hewett, B. Lillie, and T. G. Rizzo, Warped higgsless models with ir-brane kinetic terms, JHEP 05 (2004) 015, hep-ph/0403300].

[15] R. Barbieri, A. Pomarol, R. Rattazzi and A. Strumia, Electroweak symmetry breaking after LEP1 and LEP2, Nucl. Phys. B 703 (2004) 127 [arXiv:hep-ph/0405040].

[16] J. L. Hewett, B. Lillie, and T. G. Rizzo, Monte carlo exploration of warped higgsless models, JHEP 10 (2004) 014, hep-ph/0407059].

[17] R. Foadi, S. Gopalakrishna, and C. Schmidt, Higgsless electroweak symmetry breaking from theory space, JHEP 03 (2004) 042, hep-ph/0312324.

[18] J. Hirn and J. Stern, Eur. Phys. J. C 34, 447 (2004) [arXiv:hep-ph/0401032].

[19] R. Casalbuoni, S. De Curtis and D. Dominici, Moose models with vanishing S parameter, Phys. Rev. D 70 (2004) 055010 [arXiv:hep-ph/0405188].

[20] R. S. Chivukula, E. H. Simmons, H. J. He, M. Kurachi and M. Tanabashi, The structure of corrections to electroweak interactions in Higgsless models, Phys. Rev. D 70 (2004) 075008 [arXiv:hep-ph/0406077].

[21] M. Perelstein, Gauge-assisted technicolor?, JHEP 10 (2004) 010, hep-ph/0408072].

[22] R. S. Chivukula, E. H. Simmons, H.-J. He, M. Kurachi, and M. Tanabashi, Universal non-oblique corrections in higgsless models and beyond, Phys. Lett. B603 (2004) 210-218, hep-ph/0408262.

[23] H. Georgi, Fun with Higgsless theories, Phys. Rev. D 71, 015016 (2005) [arXiv:hep-ph/0408067].

[24] R. Sekhar Chivukula, E. H. Simmons, H. J. He, M. Kurachi and M. Tanabashi, Electroweak corrections and unitarity in linear moose models, Phys. Rev. D 71 (2005) 035007 [arXiv:hep-ph/0410154].

[25] N. Arkani-Hamed, A. G. Cohen, and H. Georgi, (de)constructing dimensions, Phys. Rev. Lett. 86 (2001) 4757-4761, hep-th/0104005.

[26] C. T. Hill, S. Pokorski, and J. Wang, Gauge invariant effective lagrangian for kaluza-klein modes, Phys. Rev. D64 (2001) 105005, hep-th/0104035. 
[27] M. E. Peskin and T. Takeuchi, Estimation of oblique electroweak corrections, Phys. Rev. D46 (1992) 381-409.

[28] G. Altarelli and R. Barbieri, Vacuum polarization effects of new physics on electroweak processes, Phys. Lett. B253 (1991) 161-167.

[29] G. Altarelli, R. Barbieri, and S. Jadach, Toward a model independent analysis of electroweak data, Nucl. Phys. B369 (1992) 3-32.

[30] H. Georgi, A tool kit for builders of composite models, Nucl. Phys. B266 (1986) 274.

[31] R. S. Chivukula, H.-J. He, J. Howard, and E. H. Simmons, The structure of electroweak corrections due to extended gauge symmetries, Phys. Rev. D69 (2004) 015009, hep-ph/0307209.

[32] R. Casalbuoni, S. De Curtis, D. Dominici, and R. Gatto, Effective weak interaction theory with possible new vector resonance from a strong higgs sector, Phys. Lett. B155 (1985) 95.

[33] R. Casalbuoni et. al., Degenerate bess model: The possibility of a low energy strong electroweak sector, Phys. Rev. D53 (1996) 5201-5221, hep-ph/9510431.

[34] M. Bando, T. Kugo, S. Uehara, K. Yamawaki, and T. Yanagida, Is rho meson a dynamical gauge boson of hidden local symmetry?, Phys. Rev. Lett. 54 (1985) 1215.

[35] M. Bando, T. Kugo, and K. Yamawaki, On the vector mesons as dynamical gauge bosons of hidden local symmetries, Nucl. Phys. B259 (1985) 493.

[36] M. Bando, T. Fujiwara, and K. Yamawaki, Generalized hidden local symmetry and the a1 meson, Prog. Theor. Phys. 79 (1988) 1140.

[37] M. Bando, T. Kugo, and K. Yamawaki, Nonlinear realization and hidden local symmetries, Phys. Rept. 164 (1988) 217-314.

[38] M. Harada and K. Yamawaki, Hidden local symmetry at loop: A new perspective of composite gauge boson and chiral phase transition, Phys. Rept. 381 (2003) 1-233, hep-ph/0302103.

[39] G. Cacciapaglia, C. Csaki, C. Grojean and J. Terning, Curing the ills of Higgsless models: The $S$ parameter and unitarity, Phys. Rev. D 71 (2005) 035015 [arXiv:hep-ph/0409126].

[40] R. Foadi, S. Gopalakrishna and C. Schmidt, Effects of fermion localization in Higgsless theories and electroweak constraints, Phys. Lett. B 606 (2005) 157 [arXiv:hep-ph/0409266].

[41] R. S. Chivukula, E. H. Simmons, H. J. He, M. Kurachi and M. Tanabashi, Deconstructed Higgsless models with one-site delocalization hep-ph/0502162.

[42] R. Casalbuoni, S. De Curtis, D. Dolce and D. Dominici, Playing with fermion couplings in Higgsless models hep-ph/0502209.

[43] R. S. Chivukula, E. H. Simmons, H.-J. He, M. Kurachi, and M. Tanabashi, to be published.

[44] H.-C. Cheng, C. T. Hill, and J. Wang, Dynamical electroweak breaking and latticized extra dimensions, Phys. Rev. D64 (2001) 095003, hep-ph/0105323. 